\title{
A Method of Controlling Gantry Crane Based on Wireless Communication
}

\author{
JING Dongxu $u^{1, a}$
}

${ }^{I}$ Tongliao Vocational College, Tongliao, 028000.

ajdxtvc@163.com

\begin{abstract}
It is introduced that the operator uses radio frequency technology to communicate with gantry cranes gather relevant data and control their work. Frequency communication system consists of two parts: one part is mounted on the crane to collect working and the environmental data of crane; another part of the manual is controlled by the operator to keep the crane working and view in real time of the operating parameters of the crane. The two parts can be configured to the same address to prevent interference from the outside world and the same kind of equipment. The distance of Frequency communication can transmit information as large as $100 \mathrm{~m}$. The operator can control the equipment on the ground instead of sitting in the cab of $10 \mathrm{~m}$ high. The site has been completed experiments in Xinzheng city, which shows that the method is safe and reliable.
\end{abstract}

Keywords: Wireless communication, Crane control, RF technology, Distance transmission, SPI Interface

Since entering the 21st century, radio frequency technology has received more and more attention [1]. Cranes are special equipment, and the safety of crane equipment is related to the national economy and people's livelihood. If an accident occurs, the consequences will be inestimable. The crane industry belongs to the machinery industry, but now most crane industries are in a non-automated or semi-automated mode. How to reform, innovate, and upgrade the traditional machinery industry into a new industry of fully automated control is a big problem it faces. It is of great significance to apply video technology to the traditional machinery industry.

This paper proposes to use wireless technology to communicate with the crane to collect information about the crane itself: speed, etc; information about the working environment of the crane: wind speed and direction, etc. And control the movement of the crane trolley, main crane up and down, electric hoist up and down、 left and right [2].

\section{HARDWARE PARTS}

As shown in Figure 1, the hardware adopts a modular design, and the design is divided into two parts, one part is a handheld device [3], which is operated on-site by a technician. The other part is the control part, which is installed on the crane to control the work of the crane and collect the working status and environmental parameters of the crane. As shown in Figure 1, the two parts communicate via radio frequency. The two parts are the same as the central processing unit and the radio frequency part.

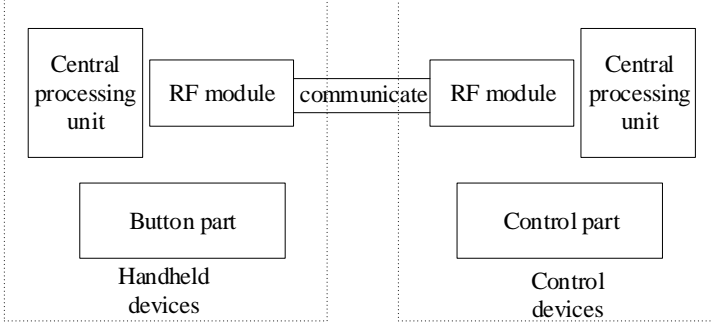

Figure 1 The total schematic diagram of hardware

\subsection{Handheld device design}

\subsubsection{Power supply section}

As shown in Figure 2, the power supply is a lithium battery that outputs a $12 \mathrm{~V}$ DC voltage, which is stabilized to $5 \mathrm{~V}$ through a 7805 voltage regulator tube to power the circuit. The lithium battery has a capacity of $8 \mathrm{Ah}$, which can meet the requirements of the construction process.

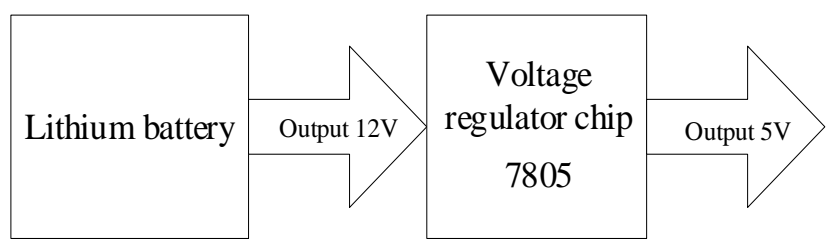

Figure 2 Schematic of power in handheld device 


\subsubsection{RF part}

As shown in Figure 3, the module adopts the original imported Norwegian nRF24LO1P chip, and the peripheral resistance-capacitance sensing devices are all imported materials, especially crystals, which use high-precision and widetemperature crystals to ensure their industrial characteristics [4] [5]. The resistors, capacitors, and inductors are packaged in 0402, and the body is very small. And the use of Murata sensing container parts, 10ppm high-precision imported crystal, the performance of the module is good. The module's open transmission distance is $50-100 \mathrm{~m}$, which can meet the needs of working in open fields. The module has an SPI interface, and the external microcontroller communicates with the module through SPI, completes the configuration and reading of the registers, and completes the sending and receiving of wireless data. The power supply voltage of the module is compatible with up to $3.6 \mathrm{~V}$, which is connected in series with a $1 \mathrm{~K}$ resistor when the microcontroller communicates. Metal objects that have a significant effect on the antenna cannot be placed near the antenna of the single-chip microcomputer; the shell cannot be made of metal material, which will shorten the distance.

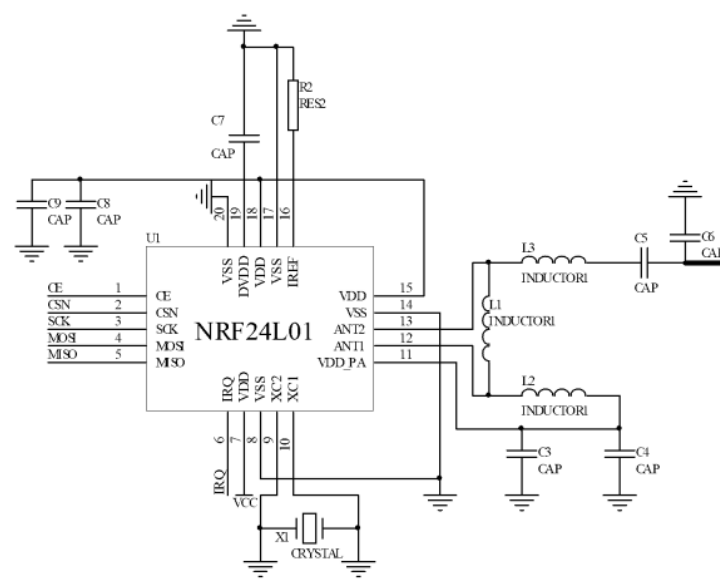

Figure 3 Schematic of RF circuit

\subsubsection{Central Processing Unit}

The one-chip computer uses ATMEGA8, this chip has strong anti-interference ability, rich peripherals, internal $4 \mathrm{M}$ crystal oscillator and internal reset, internal 512-byte EEPROM, which can store some operating parameters that need to be saved in adjustment process s setting process and power is off [6].

\subsection{Control equipment}

\subsubsection{Power supply section}

The handheld part of the power supply is converted from an industrial $380 \mathrm{~V}$ power supply to $5 \mathrm{~V}$ to supply power to the microcontroller and its peripheral circuits. The power supply adopts modules produced by Shanghai BoPai. The module has complete output overvoltage and overload protection functions; conduction can pass CLASS $\mathrm{B}$; it has strong anti-surge and group pulse interference capability; efficiency is greater than $5 \%$.

\subsubsection{Control part}

Information collection element: lifting weight limiter, lifting height limiter, descending depth limiter, running stroke limiter, deflection limiter, interlock protection safety device (door limit, operation interlock between institutions), anti-wind and anti-skid device, anemometer device, rotation limiter, anti-collision device of the same or different track running mechanism, overspeed protection device, safety limit of power supply cable drum, lifting mechanism brake.

Monitoring items: lifting height, descent depth, running stroke, cart running deflection, wind speed, safety distance of the same or different track running mechanism, operating instructions, working time, accumulated working time, each working cycle, lifting mechanism brake Status, anti-wind and anti-skid status, interlocking protection (door limit, interlocking operation between institutions), power supply cable reel status.

As shown in Figure 4, the Input on the left is connected to the I/O pins of the microcontroller, and the Output on the right is connected to the sensor port to be controlled. Control the sensor to work when the microcontroller outputs low level. On the contrary, the sensor does not work.

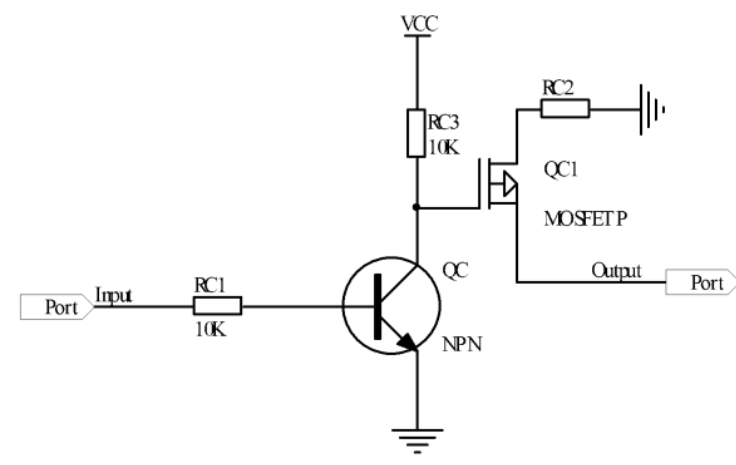

Figure 4 Diagram of controlling external sensor circuit 


\section{SOFTWARE PART}

The software is divided into two parts, one is the handheld device, and the other is the control part. The two parts must be configured with the same address when they leave the factory, and they can work together if the addresses are the same. There may be more than one crane on the same construction site, so you can prevent interference by configuring different addresses.

The one-chip computer communicates with the radio frequency module through the SPI interface. The single-chip microcomputer adopts the analog SPI interface, which can save the IO pins of the single-chip microcomputer.

\subsection{Handheld equipment}

To control the crane to start, work and stop, use handheld equipment to communicate with the crane through radio frequency technology. As shown in Figure 5, pressing the corresponding work button of the handheld device, the handheld device sends a control command, and when the feedback from the control device is received, it stops sending the command, and the handshake is successful. If the feedback signal is not received, it means that there is a problem with the control equipment, and the control command must be sent a second time. If there is no feedback signal at this time, the control command will be stopped and the error signal light will be on.

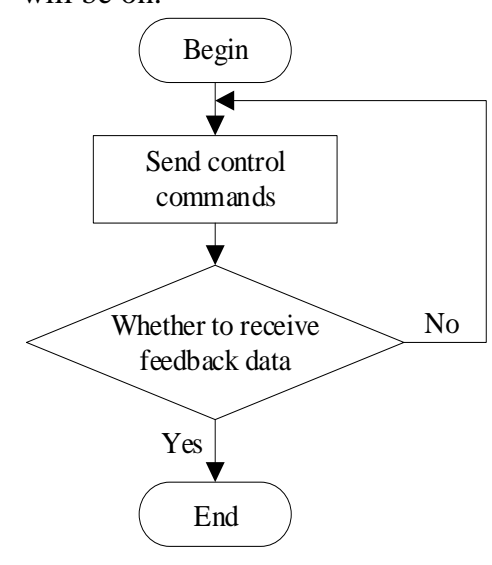

Figure 5 Program flow handheld equipment

The sent data is 8 bytes in hexadecima: AA, data length, sensor code, address, control sensor code, control sensor action, CRC check high byte, CRC check low byte.

\subsection{Control part}

As shown in Figure 6, after receiving the control signal, judge whether the address matches or not. If the address does not match the machine, it will not respond. When it is judged that the address is consistent with this machine, it will reply the message to the handheld device and then control the crane movement.

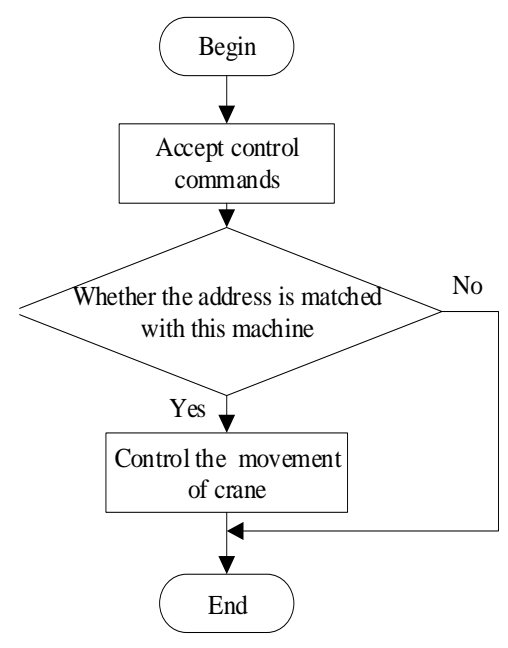

Figure 6 Program flow of controlling device

The sent data is 6 bytes in hexadecimal: 55, data length, sensor code, address, 01, CRC check high byte, CRC check low byte。

\section{EXPERIMENTAL RESULTS}

The shop inspection test has been completed in the laboratory. After completion in the laboratory, it is applied to Xinzheng crane equipment [7]. The measurement data is shown in Table 1.

Tab.1 Crane monitoring parameters

\begin{tabular}{cc}
\hline Monitoring type & Numerical value \\
Lifting height & $5 \mathrm{~m}$ \\
Wind speed & $1.5 \mathrm{~m} / \mathrm{s}$ \\
Wind direction & Northwest \\
State of Lifting & Stop \\
mechanism brake & $1 \mathrm{~m} / \mathrm{s}$ \\
Travel speed of cart & \\
Cumulative working & 20 hours \\
time & \\
\hline
\end{tabular}

\section{CONCLUSIONS}

It can be seen from the results of laboratory experiments and field experiments that the radio frequency system has the following advantages:

1 Long transmission distance, the working environment of cranes is mostly in the field or open indoor, and the transmission distance can reach $100 \mathrm{~m}$.

2 The staff can control the crane to work on the ground. In the past, the driver's cab of the crane was at an altitude of about $10 \mathrm{~m}$, which was very 
inconvenient for the staff to work. With this radio frequency system, the staff can operate freely.

3 Crane equipment manufacturing belongs to the machinery industry. Both manufacturing and manipulation are done manually, and there is no way to monitor and summarize the working parameters of many cranes. Now with this radio frequency system, the crane can be fully automated. It is easy for the staff to monitor the working environment of the crane on site and find abnormalities in the construction process.

With a system based on radio frequency technology, manpower and material resources are saved, and the safety factor in the construction process is increased.

\section{REFERENCES}

[1] Wang Jinchuan, Qin Zhen, Han Yu. Development status and application of radio frequency technology $[\mathrm{J}]$. Application of Electronic Technology, 2007, 33(7): 5-11.

[2] Tan Xiaodong, Li Zhong, Li Baoliang. Railway crane electrical control system based on PLC control technology [J]. Journal of Dalian Jiaotong University, 2009, 30(5): 15-18.

[3] Cao Lifeng, Hu Jinping, Cao Guangzhi. Design of input signal filter for crane control handle [J]. Manufacturing Information, 2013, (6): 111-113.

[4] Zhang Yemao, Zhang Jiangong, Zhang Guangzhou, etc. Design of wireless communication DC electric field measurement system based on nRF905 module [J]. High Voltage Technology, 2012, 38(11): 2869-2874.

[5] Mo Xiaojin, Zhou Yan. Temperature and humidity remote monitoring system based on radio frequency technology and wireless network [J]. Journal of Sensor Technology, 2011, 24(10): 1501-1505.

[6] Zhou Weifang, Rong Yue. Design of long-distance transmission controller based on single-chip microcomputer $[\mathrm{J}]$. China New Technology and New Products, 2012, (8): 22-23.

[7] Zhou Yuanhui. Research and implementation of the control system of the straw bale grab crane [D]. Xi'an: Xi'an University of Science and Technology, 2008.

[8] H. Li, P. Xu, et. al., Low-voltage and fast-response $\mathrm{SnO} 2$ nanotubes/perovskite heterostructure photodetector, Nanotechnology, 32(2021)375202.
[9] Wang, Y., Wang, W., Yang, X., et. al., ITGA8 positive cells in the conventional outflow tissue exhibit Schlemm's canal endothelial cell properties, Life Sciences, 278(2021)119564.

[10] P. Xu, J. Cao, et. al., Quantum chemical study on the adsorption of megazol drug on the pristine BC3 nanosheet, Supramolecular Chemistry, 33(2021)63-69.

[11] J. He, X. Liu,et al. High Annealing Stability of InAlZnO Nanofiber Field-Effect Transistors with Improved Morphology by Al Doping. Journal of Physical Chemistry Letters, 12.4(2021)1339-1345.

[12] H. Zu, Y. Chang, H. Li, et. al., Modulating the Transport Properties of Metal Oxide Nanofibers Transistors by Controlling the Grain Size. IEEE Electron Device Letters. 42.6(2021)855-858.

[13] D. Liu, B. Yu, M. Liao, et. al., Self-Powered and Broadband Lead-Free Inorganic Perovskite Photodetector with High Stability, ACS Applied Materials \& Interfaces, 12.27(2020) 30530- 30537. 